\title{
Two-Stage Flexor Pollicis Longus Tendon Reconstruction Using Pedicled Palmaris Longus Tendon Graft
}

\author{
Ö. F. Kümbüloğlu¹, F. Canşah Barışhan², H. Mustafa Özdemir² \\ 1 Division of Hand Surgery, Department of Orthopaedic and Traumatology, Şişli Hamidiye Etfal Training and \\ Research Hospital, Şişli, Istanbul, Turkey \\ 2 Department of Orthopaedic and Traumatology, Şişli Hamidiye Etfal Training and Research Hospital, Şişli, \\ Istanbul, Turkey
}

\author{
CORRESPONDING AUTHOR: \\ Ömer Faruk Kümbüloğlu \\ Division of Hand Surgery \\ Department of Orthopaedic \\ and Traumatology \\ Şişli Hamidiye Etfal Training \\ and Research Hospital \\ Etfal Sok. Pk 34371 \\ Şişli, Istanbul, Turkey \\ E-mail: omerkumbul@gmail.com \\ DOI: \\ 10.32098/mltj.04.2021.19
}

LEVEL OF EVIDENCE: 4

\begin{abstract}
SUMMARY
Background. Free tendon grafts are frequently used in zone 2 flexor pollicis longus tendon reconstructions. However, pedicled tendon grafts have less risk of adhesion than free tendon grafts. The aim of this study was to evaluate the results of two-stage flexor pollicis longus tendon reconstruction using a pedicled palmaris longus tendon graft.

Methods. Six patients who underwent two-stage flexor pollicis longus tendon reconstruction using a pedicled palmaris longus tendon graft between 2016 and 2018 were retrospectively evaluated in this study. The mean follow-up was 17 months (range: 13-25 months). Results. In the final follow-ups, the Buck-Gramcko score was excellent in three patients, good in two patients, and fair in one patient. Mean Disabilities of the Arm, Shoulder and Hand score was 12.9 (8.3-26.7).

Conclusions. We conclude that good results can be achieved with two-stage flexor pollicis longus tendon reconstruction using a pedicled palmaris longus tendon graft. This method appears to be an alternative for flexor pollicis longus tendon reconstruction using free tendon graft.
\end{abstract}

\section{KEY WORDS}

Flexor pollicis longus tendon reconstruction; palmaris longus tendon graft; Paneva-Holevich; pedicled tendon graft; two-stage flexor tendon reconstruction.

\section{BACKGROUND}

The reconstruction of the zone 2 flexor tendon divisions using a tendon graft has been performed for many years. Hunter and Salisbury defined the two-stage reconstruction in 1971 (1). Since then, it has been used in cases where single-stage flexor tendon reconstruction was not appropriate (2-4). In 1972, Kessler reported the results of two-stage flexor digitorum profundus (FDP) reconstruction using a pedicled flexor digitorum superficialis tendon graft together with a silicone rod (5). This technique is now called the modified Paneva-Holevich technique and is often used in FDP reconstructions (6-10). In 1978, Foucher et al. described two-stage flexor tendon reconstruction using a pedicled palmaris longus (PL) tendon graft, which was prepared in the distal part of the forearm (11). Although this technique has not found a wide range of use for FDP reconstruction, it seems to be suitable for flexor pollicis longus (FPL) reconstruction in terms of providing a pedicled tendon graft $(6,8,12)$. Currently, zone 2 FPL reconstructions are often performed with free tendon graft. However, the results obtained with this technique are not encouraging (13-15). We hypothesized that the problem with this technique is the use of free tendon graft. Because the free tendon graft needs surface neovascularization during the healing phase and this situation tends to the adhesion of the tendon graft to the surrounding tissues (16). Pedicled tendon grafts provide a great advantage in this respect. 
The aim of the present study was to evaluate the results of two-stage FPL tendon reconstruction using a pedicled PL tendon graft.

\section{MATERIALS AND METHODS}

\section{Patients and methods}

Six patients who underwent two-stage FPL tendon reconstruction using pedicled PL tendon graft between April 2016 and July 2018 were included in the present study. Only the clean-cut injuries are included to the study. The mean age of the patients was 32 years (range: 19-44 years). The study included four male patients and two female patients. The injury was in the dominant hand of one patient and in the non-dominant hands of the other patients.

While three patients were employed in various jobs, two were homemakers and one was a student. None of the patients were manual workers. All patients were injured due to cutting incidents and the primary injury site of the FPL tendon was in zone 2. None of the patients had previously undergone surgery due to this injury. All patients had missed injuries. The mean duration from injury to first stage surgery was 14 months (range 5-34).

Preoperatively, the primary complaint of four patients was loss of function in the thumb, while the primary complaint of the remaining two patients was pain in the volar aspect of the thumb interphalangeal joint.

In the final follow-ups, ranges of motion of the interphalangeal and the metacarpophalangeal joint were measured clinically with a goniometer. Static pinch force was measured with key pinch grip and second finger pulp using a pinch gauge (Baseline Pinch Gauge, Alimed Corp., Dedham, MA, USA). Results regarding active range of motion were assessed by Buck-Gramcko technique. Hand function was evaluated with Disabilities of the Arm, Shoulder, and Hand (DASH) score.

Patients were asked to rate their thumb functions as excellent, good, fair, or poor in the postoperative period.

Statistical evaluation was performed using Wilcoxon signed-rank test.

All procedures performed in our study were in accordance with the ethical standards of the Declaration of Helsinki and were approved by the local Ethics Committee (2020-1472). Informed consent was obtained from all patients involved in this study.

\section{Surgical technique}

All operations were performed under regional or general anaesthesia by application of an arm tourniquet.
As in the classical two-stage FPL reconstruction, the thumb flexor system was exposed through a Bruner incision in the first stage and all the scarred tissues around the flexor system were excised carefully. Nearly $1 \mathrm{~cm}$ distal attachment of the FPL tendon was preserved and the dysfunctional part of the FPL tendon was excised. All preservable pulley structures were preserved. Subsequently, the proximal end of the FPL tendon was explored by approaching with a curved incision at the volar aspect of the distal side of the forearm. The distal end of the FPL tendon was pulled into the incision site on the forearm. The silicone tendon implant was passed through the carpal tunnel and advanced to the base of the thumb (figure $1 \mathrm{~A}$ ). Subsequently, the distal end of the PL tendon was cut as distally as possible. The FPL tendon was shortened. The distal end of the FPL tendon was sutured to the distal end of the PL tendon with Pulvertaft sutures. The tenorrhaphy line remained outside the carpal tunnel (figure $1 \mathrm{~B})$. The silicone tendon implant was threaded through the pulley system to the tip of the thumb. The distal end of the implant was sutured to the surrounding soft tissue. While the tendon implant was being pulled proximally, the thumb was flexed and there was no bowstring effect (figure $1 \mathrm{C})$. Subsequently, the wound was closed. After the third postoperative day, passive range of motion exercises for the thumb were started.

The second stage was performed 3 months after the first stage. In the second stage, the distal aspect of the forearm was explored and the proximal end of the silicone tendon implant was located. Subsequently, the distal tip of the tendon implant was located by approaching the volar side of the distal thumb. Through transverse incisions made in the forearm, the PL tendon was freed and cut from the distal end of the musculotendinous junction and the proximal end of the tendon was pulled distally (figure $1 \mathbf{D}$ ). The tip of the pedicled PL tendon graft was sutured to the proximal end of the silicone tendon implant. The implant was pulled from the distal incision of the thumb, delivering the PL tendon graft to the distal phalanx. At this stage, the tension of the tendon graft was adjusted by holding the interphalangeal joint flexed at 30 degrees while the wrist was stabilised at neutral position. The PL tendon graft was temporarily fixed to the distal side of the thumb with a hypodermic needle (figure $1 \mathrm{E}$ ). Subsequently, excess tendon graft was excised. The tendon graft was fixed to the distal phalanx with a pullout suture using a button and direct suturing to the FPL stump (figure $\mathbf{1}$ F).

Postoperatively, a dorsal short arm splint was applied with the wrist in neutral position, the thumb carpometacarpal joint abducted at 30 degrees, and the metacarpophalangeal and the interphalangeal joints flexed at 30 degrees. Range of motion exercises including passive flexion and active exten- 

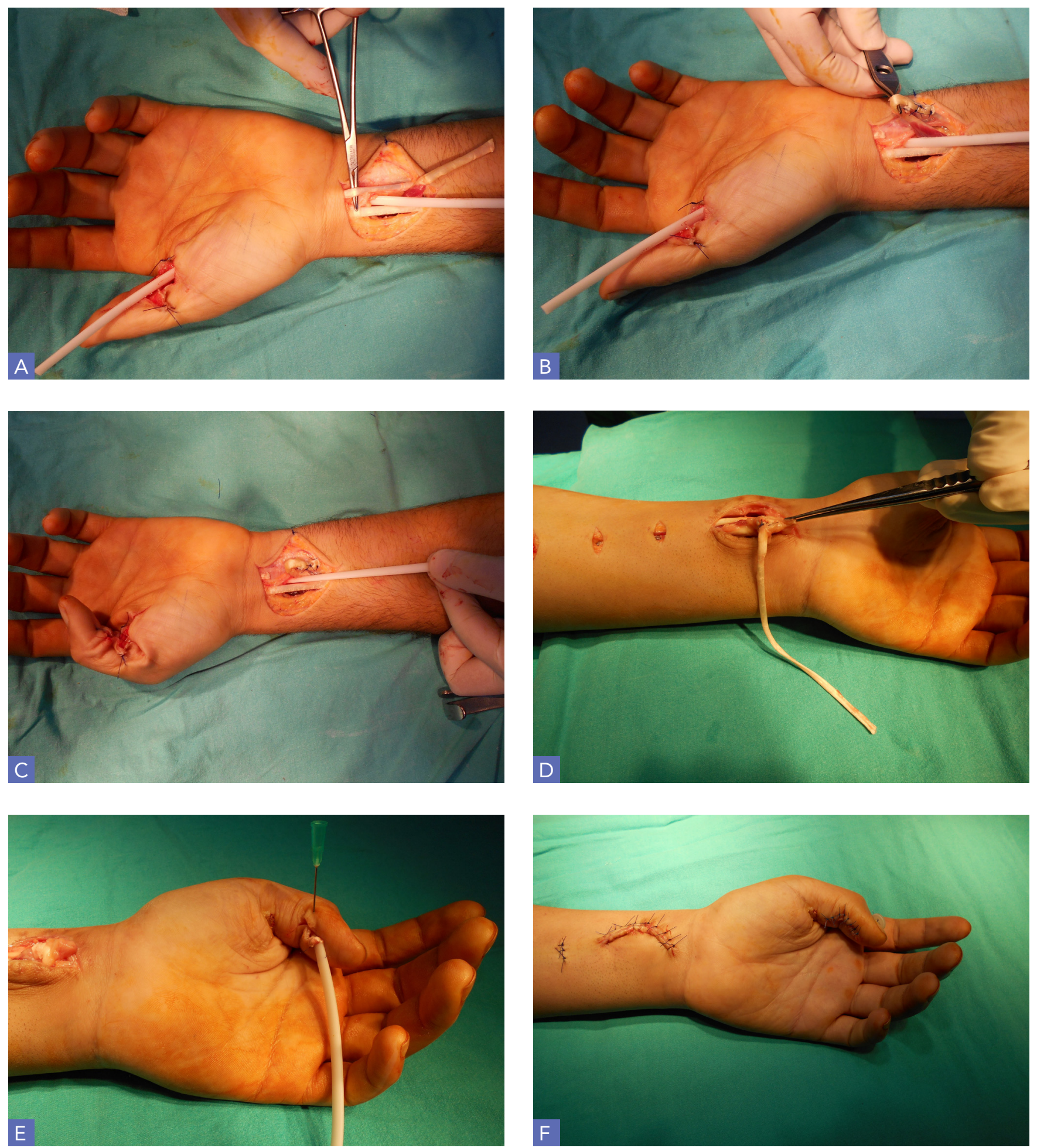

Figure 1. (A) Intraoperative photograph shows the silicone tendon implant inserted through the carpal tunnel with flexor pollicis longus (FPL) and palmaris longus (PL) tendons on its ulnar side; (B) FPL and PL tendons with their distal ends sutured together; (C) No bowstring is observed after the tendon implant is pulled proximally; (D) Pedicled PL tendon graft; (E) Adjustment of the length of the tendon graft with hypodermic needle; (F) Appearance of the thumb after pull-out suture. 
sion were instructed to be performed for six weeks postoperatively. Active thumb flexion exercises were started with the removal of the button at the end of the sixth week.

\section{RESULTS}

The mean follow-up duration was 17 months (range: 13-25 months). None of the patients developed infection or tendon rupture. No additional surgical intervention was performed in any patient.

Results are summarised in table $\mathbf{I}$ and see figure 2.

After reconstruction, mean extension deficits of 14 degrees (range: 0-25 degrees) and 11 degrees (range: 0-20 degrees) were observed in the interphalangeal joint and the meta- carpophalangeal joint, respectively. The range of motion of the interphalangeal and the metacarpophalangeal joint on the operated side was significantly lower than that on the contralateral side $(\mathrm{P}<0.05)$. The values of key pinch strength and pinch grip strength for the index finger were significantly lower on the operated side than on the contralateral side $(\mathrm{P}<0.05)$. The Buck-Gramcko score was excellent in three patients, good in two patients, and fair in one patient. Mean DASH score was 12.9 (8.3-26.7).

Patients subjectively evaluated their thumb functions postoperatively. Five patients rated their functions as good, while one patient rated the functions as fair. Two patients with main complaint of pain at admission stated that the pain was relieved in the postoperative period. Three patients who

Table I. Results of the flexor pollicis longus tendon reconstruction.

\begin{tabular}{lccc}
\hline & Mean & Range & $\%$ of contralateral side mean value \\
\hline Active Flexion IP & $59^{\circ}$ & $35^{\circ}-75^{\circ}$ & $81.9 \%$ \\
\hline Active ROM IP & $45^{\circ}$ & $20^{\circ}-65^{\circ}$ & $62.5 \%$ \\
\hline Active ROM MCP & $44^{\circ}$ & $25^{\circ}-60^{\circ}$ & $69.8 \%$ \\
\hline Active ROM IP + MCP & $89^{\circ}$ & $60^{\circ}-120^{\circ}$ & $65.9 \%$ \\
\hline Pinch strength to the index finger $(\mathrm{kg})$ & 5.5 & $2.3-8.8$ & $70.5 \%$ \\
\hline Key pinch strength $(\mathrm{kg})$ & 6.9 & $3.8-12.1$ & $62.7 \%$ \\
\hline
\end{tabular}

IP: interphalangeal joint; MCP: metacarpophalangeal joint; ROM: range of motion.
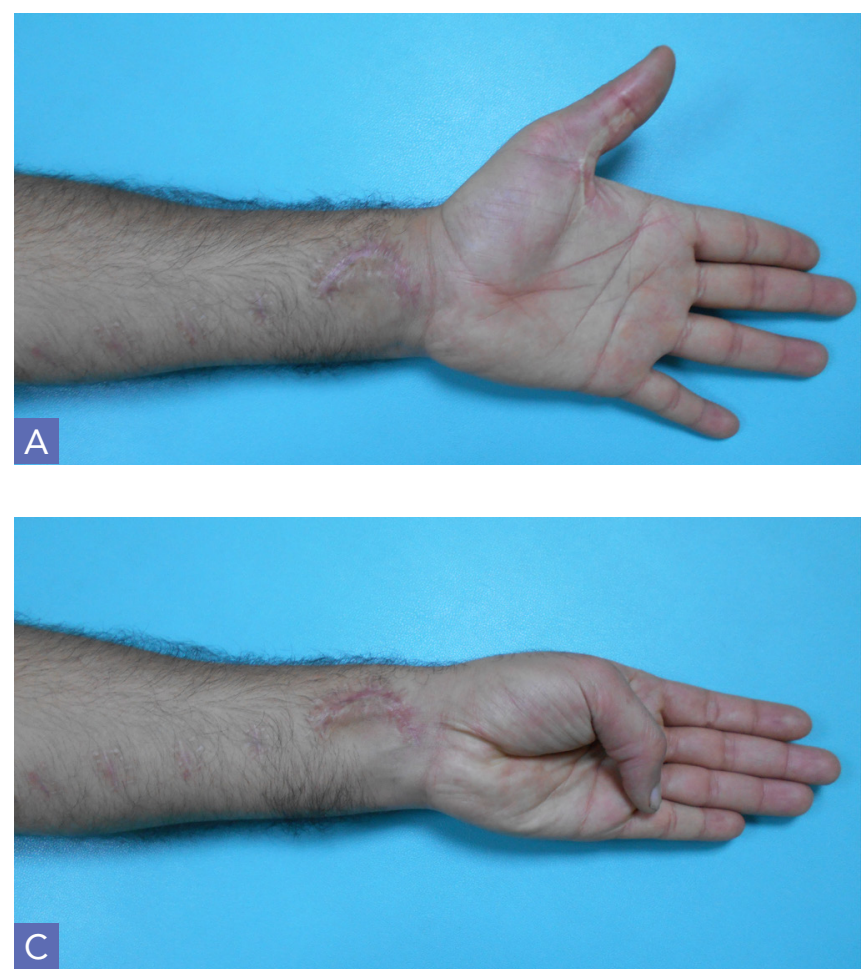

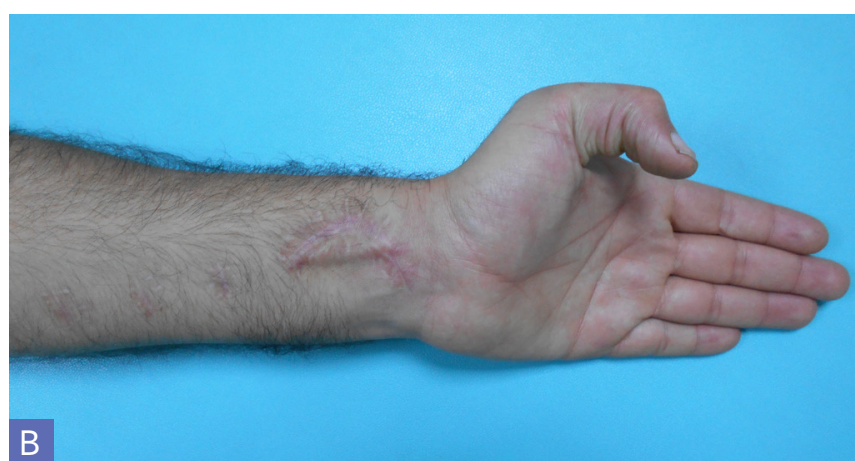

Figure $2 \mathrm{~A}-\mathrm{C}$. Thumb range of motions in the same patient at the sixth postoperative month. 
were employed in jobs were able to return to their previous jobs at a mean duration of 13 weeks (range: $12-15$ weeks) after the second stage.

\section{DISCUSSION}

Successful results are frequently obtained with primary repairs of zone 2 FPL divisions (17). If primary repair is not possible or fails, tendon grafting is the common treatment method. Unfortunately the results of tendon grafting are generally not as successful as primary repairs (13). According to the Buck-Gramcko score, we achieved excellent and good results in five of the six patients that we performed FPL tendon reconstruction with pedicled PL tendon graft. The main limitation of this study was the small number of patients. Currently, most of the FPL divisions are successfully diagnosed and treated in the acute period, consequently limiting the number of patients. All the patients in the present study were injured by cutting incidents. However, FPL divisions may be associated not only with this type of injury, but also with high-energy injuries. Patients who had undergone unsuccessful repair of the FPL division previously are also the candidates for FPL reconstruction. However, all patients in the present study had missed injuries. Hence, the patients in the present study had better prognoses.

Single stage FPL reconstructions are generally considered appropriate for patients with intact tendon sheath and mobile joints (Boyes grade 1), as is the case with single stage FDP reconstructions (18-20). There is a key factor distinguishing the FPL tendon from the FDP tendon, which is the path between the distal phalanx and the forearm. There are very sharp angulations along this path that creats a suitable ground for adhesion of the free tendon graft. While the proximal tenorrhaphy can be performed to the palmar of the hand in most FDP reconstructions, in FPL reconstructions, proximal tenorrhaphy is frequently performed in the distal forearm, which causes the free tendon graft to pass through the carpal tunnel, requiring the graft to be longer. For these reasons, we believe it to be beneficial to create a pseudosheath formation with two-stage reconstruction in all FPL reconstructions. Two-stage reconstruction also gives us the chance to use a pedicled tendon graft. The waiting period between the two stages is a disadvantage of this method, but this time is not considered as a problem by patients who have learned the value of the thumb function.

Functional return may be unsatisfactory in patients undergoing a two-stage FPL reconstruction using free tendon grafts. Therefore some authors recommended considering interphalangeal joint arthrodesis instead of FPL reconstruction $(14,15,21)$. However, single interphalangeal arthrodesis in patients with chronic FPL injury has been reported to cause a significant loss of pinch strength. Therefore, FPL reconstruction is critical to maintain the pinch force (22). In light of these reports, it is necessary to obtain better results in two stage reconstruction.

Free tendon grafts begin to vascularise on the twenty-first postoperative day and the number of surviving cells is limited (23). Surface neovascularisation plays a role in the healing phase of all free tendon grafts irrespective of its extent. This tends to result in adhesion to the surrounding tissues (16). However, healing process of pedicled tendon grafts differs from that of free tendon grafts. Pedicled tendon grafts that were separated from their proximal origin 5 weeks after the first stage have been shown to preserve their vascularisation (24). We did not use free tendon graft in this technique. Thus, we tried to overcome the problem of adhesion.

Pain is expected in the unrepaired zone 2 flexor tendon divisions, especially in the palm. This pain limits itself within a few months, even if the patient is not treated. In our study, two patients who suffered from pain in the volar aspect of the thumb interphalangeal joint preoperatively reported no pain at the final follow-up. We assumed that this pain was caused by the use of the volar side of the thumb interphalangeal joint to increase the grasping strength while holding the objects. Since active interphalangeal joint flexion was achieved, patients' complaints were relieved, as the load transfer was performed by the pulp of the thumb.

Irrespective of the treatment (primary repair or reconstruction), a functional pulley system is indispensable in flexor tendon surgery. Especially, A1 pulley is important for the FPL function (25-27). Least possible damage to the pulley system directly increases the functional gain. However, this never be considered as, allowing a tissue in the flexor system that will prevent tendon slipping. At the end of the first stage, the pulley system should be re-evaluated with movement of the tendon prosthesis and if necessary, pulley reconstruction combined with scar tissue excision should not be avoided.

Adjusting the length of the tendon graft in FPL and FDP tendon reconstructions is one of the most important challenges associated with the surgery. Impossibility of adjusting the length of the graft using the proximal tenorrhaphy area appears to be an important technical difficulty in this technique. Keeping the graft too tight or too loose may cause quadriga syndrome and lumbrical plus finger problems in FDP reconstructions. However, the complication interval is narrower, since these problems are not encountered in FPL reconstructions $(6,28)$.

In this technique, some problems have still not been overcome. One of these problems is the mismatch between the cross sectional area (CSA) of the tendon graft and the CSA of the reconstructed tendon. In flexor tendon reconstruc- 
tions, the CSA of the tendon graft is always intended to be close to that of the tendon to be reconstructed (29). Therefore, the CSA of the PL tendon being considerably lower than the CSA of the FPL tendon is considered a disadvantage $(29,30)$. The CSA of the flexor carpi radialis (FCR) tendon is very close to that of the FPL tendon (30). Therefore, it may be appropriate to use a part of the FCR tendon as a pedicled tendon graft in this technique. However, it should be taken into account that the maximum obtained length of the FCR tendon graft will be shorter the length of the PL tendon graft. It may be preferable to perform the proximal tenorrhaphy using modified Kessler technique instead of the Pulvertaft technique to provide sufficient tendon graft length. The use of pedicled FCR tendon graft may also an option for patients without a PL tendon.

Many studies have suggested that the use of intrasynovial tendons as grafts, especially in the pulley system, is more appropriate than the use of extrasynovial tendons (16, 31,32). The use of PL tendon, which is an extrasynovial tendon, is another disadvantage of this technique.

\section{CONCLUSIONS}

Over the years, the unsatisfactory results we obtained with zone 2 FPL reconstructions using free tendon graft led us to seek a different method. As a result, we abandoned FPL reconstruction with a free tendon graft because we consid-

\section{REFERENCES}

1. Hunter JM, Salisbury RE. Flexor-tendon reconstruction in severely damaged hands. A two-stage procedure using s silicone-dacron reinforced gliding prosthesis prior to tendon grafting. J Bone Joint Surg Am 1971;53:829-58.

2. Amadio PC, Wood MB, Cooney WP, Bogard SD. Staged flexor tendon reconstruction in the fingers and hand. J Hand Surg Am 1988;13:559-62.

3. Goldfarb CA, Gelberman RH, Boyer MI. Flexor tendon reconstruction: current concepts and techniques. J Am Soc Surg Hand 2005;5:123-30.

4. Wehbé MA, Mawr B, Hunter JM, Schneider LH, Goodwyn BL. Two-stage flexor-tendon reconstruction. Ten-year experience. J Bone Joint Surg Am 1986;68:752-63.

5. Kessler FB. Use of a pedicled tendon transfer with a silicone rod in complicated secondary flexor tendon repairs. Plas Recon Surg 1972;49:439-43.

6. Beris AE, Darlis NA, Korompilias AV, Vekris MD, Mitsionis GI, Soucasos PN. Two-stage flexor tendon reconstruction in zone II using a silicone rod and pedicled intrasynovial graft. J Hand Surg Am 2003;28:652-60.

7. Naam NH. Staged flexor tendon reconstruction using pedicled tendon graft from the flexor digitorum superficialis. J Hand Surg Am 1997;22:323-7. er that these poor results were due to the use of an avascular tendon graft. We apply a two-stage reconstruction with pedicled tendon graft to each patient who is deemed suitable for either a single or two-stage reconstruction by classical evaluation. This method is technically more difficult and has to be done in two stages. However, the FPL function we are trying to achieve is more valuable than these disadvantages. Good results can be obtained with two-stage FPL tendon reconstruction using a pedicled PL tendon graft. This method appears to be an alternative for FPL tendon reconstruction using a free tendon graft.

\section{ETHICS}

This study was approved by the local Ethics Committee of Şişli Etfal Teaching and Research Hospital and was conducted in accordance with the ethical standards of the Declaration of Helsinki.

\section{INFORMED CONSENT}

Informed consent was obtained from all patients involved in this study.

\section{CONFLICT OF INTERESTS}

The authors declare that they have no conflict of interests.
8. O'Shea K, Wolfe SW. Two-stage reconstruction with the modified Paneva-Holevich technique. Hand Clin 2013;29:223-33.

9. Paneva-Holevich E. Two-stage plasty in flexor tendon injuries of fingers within the digital synovial sheath. Acta Chir Plast 1965;7:112-24.

10. Paneva-Holevich E. Two-stage tenoplasty in injury of the flexor tendons of the hand. J Bone Joint Surg Am 1969;51:21-32.

11. Foucher G, Merle M, Sibilly A, Michon J. Flexor tendon grafting. The use of a modified Hunter's technique (author's transl). Rev Chir Orthop Reparatrice Appar Mot 1978;64:703-5.

12. Pauchard N, Pedeutour B, Dautel G. Reconstruction par greffe des tendons fléchisseurs. Chir Main 2014;33:S58-S71.

13. Moore T, Anderson B, Seiler III JG. Flexor tendon reconstruction. J Hand Surg Br 2010;35(6):1025-30.

14. Frakking TG, Depuydt KP, Kon M, Werker PM. Retrospective outcome analysis of staged flexor tendon reconstruction. J Hand Surg Br 2000;25:168-74.

15. Unglaub F, Bultmann C, Reiter A, Hahn P. Two-staged reconstruction of the flexor pollicis longus tendon. J Hand Surg Br 2006;31:432-5. 
16. Wong R, Alam N, McGrouther AD, Wong JKF. Tendon grafts: their natural history, biology and future development. J Hand Surg Eur 2015;40:669-81.

17. Sirotakova M, Elliot D. Early active mobilization of primary repairs of the flexor pollicis longus tendon with two Kessler two-strand core sutures and a strengthened circumferential suture. J Hand Surg Br 2004;29(6):531-5.

18. Aydin A, Topalan M, Mezdeği A, et al. Fleksör tendon yaralanmalarinda tek seansli fleksör tendoplasti [Singlestage flexor tendoplasty in the treatment of flexor tendon injuries]. Acta Orthop Traumatol Turc 2004;38(1):54-9.

19. Boyes JH. Flexor-tendon grafts in the fingers and thumb: An evaluation of end results. JBJS 1950;32(3):489-531.

20. Derby BM, Wilhelmi BJ, Zook EG, Neumeister MW. Flexor tendon reconstruction. Clin Plast Surg 2011;38(4):607-19.

21. Bickert B, Kremer T, Kneser U. Sekundäre Sehnenrekonstruktionen am Daumen. Unfallchirurg 2016;119:986-92.

22. Goetz TJ, Costa JA, Slobogean G, Patel S, Mulpuri K, Travlos A. Contribution of flexor pollicis longus to pinch strength: an in vivo study. J Hand Surg Am 2012;37:2304-9.

23. Alam N, McGrouther DA, Wong JK. The cellular biology of tendon grafting. J Hand Surg Eur 2014;39:79-92.

24. Chaplin DM. The vascular anatomy within normal tendons, divided tendons, free tendon grafts and pedicle tendon grafts in rabbits: a microradioangiographic study. J Bone Joint Surg Br 1973;55:369-89.
25. Bayat A, Shaaban H, Giakas G, Lees VC. The pulley system of the thumb: anatomic and biomechanical study. J Hand Surg Am 2002;27:628-35.

26. Schneider AD, Srinivas M, Hijji FY, Jerkins D, Wimalawansa SM. Anatomic Considerations and Reconstruction of the Thumb Flexor Pulley System. Tech Hand Up Extrem Surg 2019;23:191-5.

27. Zissimos AG, Szabo RM, Yinger KE, Sharkey NA. Biomechanics of the thumb flexor pulley system. J Hand Surg Am 1994;19:475-9.

28. Strickland JW. Delayed treatment of flexor tendon injuries including grafting. Hand Clin 2005;21:219-43.

29. Carlson GD, Botte MJ, Josephs MS, Newton PO, Davis JL, Woo SL. Morphologic and biomechanical comparison of tendons used as free grafts. J Hand Surg Am 1993;18:76-82.

30. Cutts A, Alexander RM, Ker RF. Ratios of cross-sectional areas of muscles and their tendons in a healthy human forearm. J Anat 1991;176:133-7.

31. Gelberman RH, Seiler JG, Rosenberg AE, Heyman P, Amiel D. Intercalary flexor tendon grafts. A morphological study of intrasynovial and extrasynovial donor tendons. Scand J Plast Reconstr Surg 1992;26:257-64.

32. Seiler JG 3rd, Gelberman RH, Williams CS, et al. Autogenous flexor-tendon grafts. A biomechanical and morphological study in dogs. J Bone Joint Surg Am 1993;75:1004-14. 